\title{
Leishmania (Viannia) braziliensis em cães naturalmente infectados
}

\author{
Leishmania (Viannia) braziliensis in naturally infected dogs
}

\author{
Maria de Fátima Madeira ${ }^{1,2}$, Cláudia M. Antunes Uchôa ${ }^{3}$, Cristianni Antunes Leal' ${ }^{1}$, Roger M. Macedo Silva ${ }^{1}$, \\ Rosemere Duarte ${ }^{1}$, Ciléia M. Magalhães ${ }^{4}$ e Cathia Maria Barrientos Serra ${ }^{4}$
}

Resumo Foram estudados oito cães provenientes do Município de Maricá (RJ), com lesões sugestivas de leishmaniose tegumentar americana por métodos parasitológicos e sorológicos. Leishmania spp foi encontrada em seis cães através do cultivo in vitro. Anticorpos específicos foram detectados em seis animais pelo ELISA e em dois pela imunofluorescência indireta. Cinco isolados caninos analisados apresentaram zimodema similar a Leishmania (Viannia) braziliensis. Sugere-se que cães clinicamente suspeitos sejam acompanhados periodicamente, na tentativa de confirmar o diagnóstico da leishmaniose tegumentar canina.

Palavras-chaves: Cão. Leishmania. Diagnóstico.

Abstract Eight dogs from Maricá Municipality (RJ), with suggestive lesion of american tegumentary leishmaniasis were studied by parasitological and serological methods. Leishmania spp was found in six dogs by in vitro cultivation. Specific antibodies were detected in six dogs by ELISA and in two by indirect immunofluorescence. Five canine isolates were found to belong to the same zymodeme as Leishmania (Viannia) braziliensis. The authors suggest that clinically suspect dogs should be followed-up in an attempt to confirm the diagnostic of canine tegumentary leishmaniasis.

Key-words: Dog. Leishmania. Diagnosis.

A leishmaniose tegumentar americana (LTA) é uma zoonose amplamente distribuída no território brasileiro, ocorrendo em todas as regiões do país. Em 2000, foram notificados na região sudeste 3.059 casos humanos da doença ${ }^{3}$.

Apesar de ser uma zoonose originalmente silvestre, a LTA causada pela Leishmania (Viannia) braziliensis tem sido descrita por diversos autores ocorrendo em ambientes domésticos, tendo sido aventada a possibilidade de que animais domésticos e peridomésticos, e em especial o cão, estariam funcionando como importantes fontes de infecção nesse locais ${ }^{2}{ }^{13}$. Vários questionamentos ainda permanecem quanto ao verdadeiro papel do cão nessa nova adaptação do ciclo de transmissão ${ }^{16}$.

Pouco também se conhece sobre os aspectos clínicos, parasitológicos e imunológicos do curso da infecção pela $L$. braziliensis em cães, sendo portanto, necessários mais estudos visando elucidar esses aspectos, que contribuirão não só para o diagnóstico precoce dos casos caninos bem como para o esclarecimento do papel dessa espécie animal na epidemiologia da LTA.

O presente trabalho teve como objetivo estudar casos de cães com lesão sugestiva de LTA provenientes do Município de Maricá (Rio de Janeiro), descrevendose a apresentação das lesões e correlacionando-as com os resultados parasitológicos e sorológicos.

\footnotetext{
1. Departamento de Ciências Biológicas da Escola Nacional de Saúde Pública da Fundação Oswaldo Cruz, Rio de Janeiro, RJ. 2. Departamento de MicroImuno-Parasitologia do Instituto de Pesquisa Clínica Evandro Chagas da Fundação Oswaldo Cruz, Rio de Janeiro, RJ. 3. Departamento de Microbiologia e Parasitologia do Instituto Biomédico da Universidade Federal Fluminense, Niterói, RJ. 4. Departamento de Patologia e Clínica Veterinária da Faculdade de Veterinária da Universidade Federal Fluminense, Niterói, RJ.

Endereço para correspondência: Dra Maria de Fátima Madeira. Dept ${ }^{\circ}$ de Micro-Imuno-Parasitologia/IPCEC/FIOCRUZ. Av. Brasil 4365, 21045-900 Manguinhos, Rio de Janeiro, RJ, Brasil.

Tel: 552125984261 ramal 141; Fax: 5521 2590-9988

e-mail: fmadeira@ipec.fiocruz.br; fmadeira@ensp.fiocruz.br

Recebido para publicação em 25/7/2002

Aceito em 23/7/2003
} 


\section{MATERIAL E MÉTODOS}

Procedência dos cães. Os cães foram triados através de um inquérito casa a casa realizado nas localidades de Inoã, Itaipuaçu e Ponta Negra no Município de Maricá (Rio de Janeiro) entre 1998 e 2001. Foram examinados quanto ao estado geral de saúde e à presença de lesões sugestivas de LTA, na própria residência do proprietário. As lesões cutâneas foram consideradas sugestivas da parasitose quando ulceradas e com diâmetro igual ou maior a $5 \mathrm{~mm}$ em qualquer parte do corpo ou quando nodulares em áreas de pouco pêlo².

Em cada visita domiciliar, foi preenchida uma ficha epidemiológica na qual constavam dados da residência como proximidade com a mata, presença de outras criações e dados dos cães como origem, tempo de residência, característica de comportamento (domiciliado ou semidomiciliado), sexo, raça, idade aproximada e doença anterior ou atual.

Diagnóstico parasitológico. Das lesões sugestivas foram realizadas biópsias visando o isolamento in vitro. Da borda da lesão foi retirado um pequeno fragmento, o qual foi imerso em solução salina contendo 1000UI de penicilina G potássica (SIGMA), $100 \mu \mathrm{g}$ de estreptomicina (SIGMA) e $50 \mu \mathrm{g}$ de 5fluorocitosina (SIGMA) por mililitro. Após 24 horas a $4^{\circ} \mathrm{C}$, os fragmentos foram seccionados e semeados em meio bifásico NNN ( Novy, MacNeal e Nicolle)/Schneider's Insect Medium (SIGMA) contendo $10 \%$ de soro fetal bovino. O material foi incubado a $28^{\circ} \mathrm{C}$ por um mês, sendo realizados exames semanais a partir do $5^{\circ}$ dia.
Diagnóstico sorológico. Imuno-fluorescência indireta (IFI): a reação de imuno-fluorescência indireta, para a pesquisa de IgG, foi realizada segundo a descrição do kit de IFI para diagnóstico da leishmaniose canina (Biomanguinhos-Rio de Janeiro/FIOCRUZ/MS). Considerouse como positivas amostras que apresentaram fluorescência em diluições iguais ou maiores que 1/40.

ELISA: o método utilizado teve como princípio a metodologia indireta para pesquisa de imunoglobulinas totais de cão específico para antígenos de Leishmania (promastigotas de cepa JOF), previamente fixados em placa de poliestireno de fundo chato com 96 poços. Os soros foram diluídos a 1:20 em duplicata. A determinação da reatividade baseou-se em leituras superiores ao cut off.

Caracterização isoenzimática. Os isolados foram caracterizados pela eletroforese de enzimas (isoenzimas) empregando 5 sistemas enzimáticos: glicose-6-fosfato desidrogenase (G6PDH; E.C 1.1.1.49); glicose fosfo-isomerase (GPI; E.C.5.3.1.9); nucleotidase 1 e 2 (NH1 e NH2; E.C.3.2.2.1); fosfoglico desidrogenase (6PGDH; E.C.1.1.1.43). Todos os procedimentos foram baseados nos protocolos descritos por Momem ${ }^{10}$, sendo cada isolado comparado com o padrão de três amostras de referência. (Leishmania (Viannia) braziliensis - MHOM/ BR/75/M2903, Leishmania (Leishmania) chagasi MHOM/BR/74/PP75 e Leishmania (Leishmania) amazonensis - IFLA/BR/67/PH8).

\section{RESULTADOS}

Foram encontrados, nas localidades de Inõa, Itaipuaçu e Ponta Negra oito cães com lesões sugestivas de LTA. Todos os cães nasceram na área, tinham mais de 1 ano de idade, sete eram machos, seis domiciliados e dois semidomiciliados, sendo que apenas dois eram de raça definida (Dogue Alemão e Dachshund).

As alterações dermatológicas sugestivas de LTA estavam localizadas nas orelhas, na bolsa escrotal e no focinho, sendo, na maior parte, lesões únicas, ulceradas ou úlcero-crostosas e de evolução crônica (Figura 1).

Foi possível o isolamento de Leishmania em seis cães, após uma ou até no máximo três tentativas em pelo menos uma das lesões. $\mathrm{Na}$ ocasião do exame, quatro animais (cães de no 3 , 4,5 e 6) apresentavam mais de uma lesão. No cão no 3 foram observadas uma úlcera no focinho, duas úlceras e três pequenos nódulos na face interna da orelha esquerda. No cão no 4 foi observada uma úlcera na bolsa escrotal e outra no focinho. O cão no5 apresentou uma úlcera no focinho e múltiplas ulcerações, às vezes com presença de crostas, em ambas as faces das orelhas apresentando um padrão distinto com acometimento de região com pêlo, formato irregular e ausência de definição do bordo das ulcerações que por vezes se confluíam. Já o cão no 6 possuía úlceras na região interna de ambas as orelhas. Com exceção desses dois últimos cães, que estavam caquéticos, todos os outros estavam aparentemente saudáveis (Tabela 1).

Dos seis animais confirmados parasitologicamente, cinco procederam da localidade de Inõa e apenas um da localidade de Itaipuaçu. Destes, somente os cães no 5 e 6 foram reatores à técnica de IFI, com títulos de 1:160 e 1:40, respectivamente, sendo que no ELISA, a positividade foi verificada em cinco animais (Tabela 1).

Foram obtidos sete isolados (Tabela 1), sendo caracterizados por critérios bioquímicos as seguintes amostras: cão no 1 (úlcera na bolsa escrotal), ํㅜ 4 (úlcera na bolsa escrotal), ํㅡ 5 (pool de biopsias de ambas as orelhas ), ํo 6 (úlcera na orelha direita e úlcera na orelha esquerda).Todos esses isolados apresentaram bandas com perfis eletroforéticos semelhantes ao da amostra referência de Leishmania (V) braziliensis. 


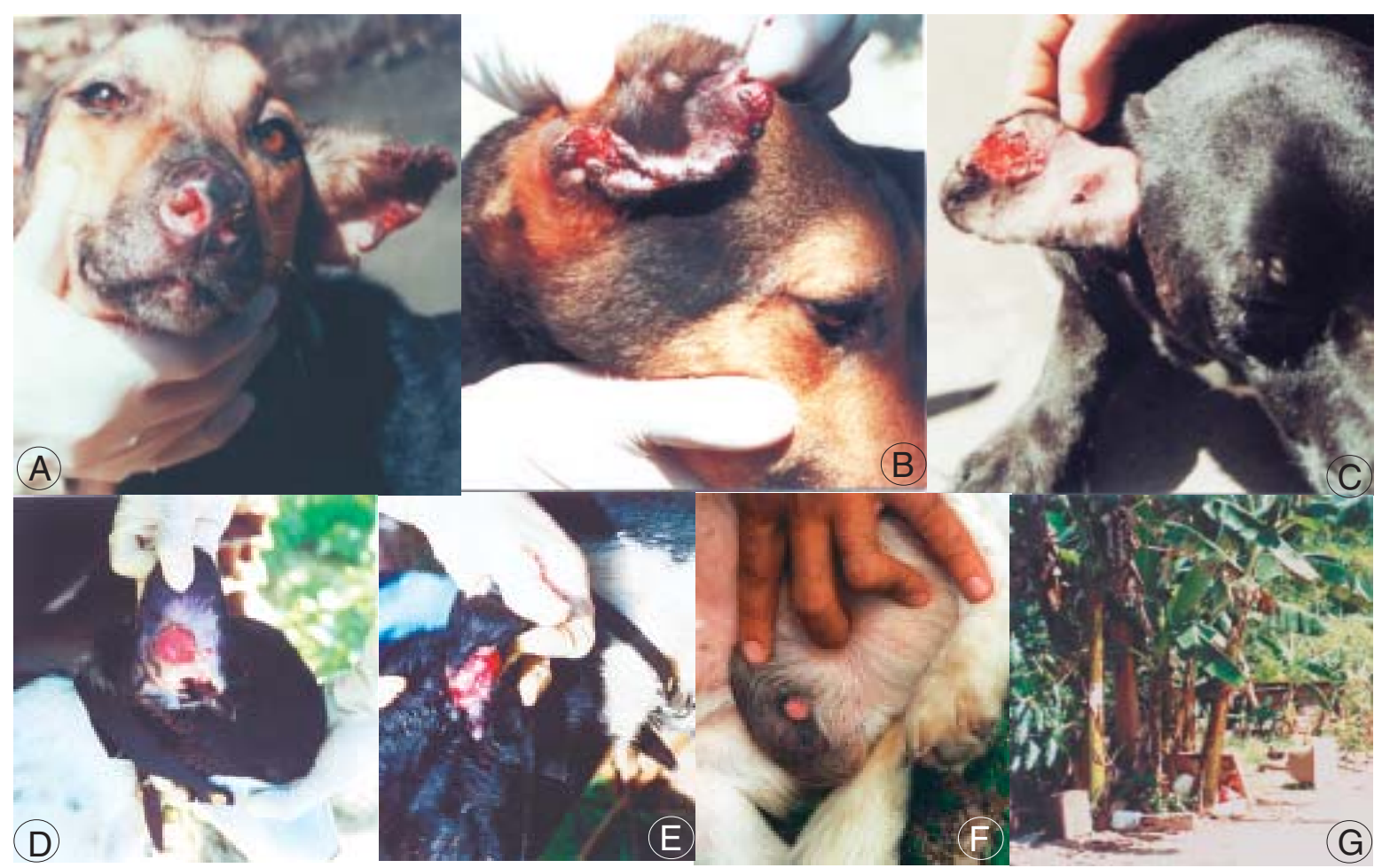

Figura 1 - Lesões tegumentares caninas provocadas por Leishmania (Viannia) braziliensis: a) lesão de mucosa do cão $n^{-}$5; b) lesão úlcero-crostosa do cão $n^{\circ} 5$; c) lesão ulcerosa do cão $n^{\circ} 3$; d/e) lesões ulcerosas do cão $n^{\circ} 6$; f) lesão ulcerosa do cão $n^{\circ} 4$; g) localidade de Inoã, Maricá, residência dos cães $n^{\circ} 5$ e 6.

Tabela 1- Resultados parasitológicos e sorológicos de oito cães com lesões sugestivas de LTA, com respectiva localização e aspecto, provenientes do Município de Marica RJ.

\begin{tabular}{|c|c|c|c|c|c|c|c|}
\hline \multicolumn{2}{|c|}{ Cão } & \multicolumn{3}{|c|}{ Lesão } & \multicolumn{3}{|c|}{ Dados laboratoriais } \\
\hline № & raça & localização & $\mathrm{n}^{\circ}$ & $\begin{array}{c}\text { aspecto } \\
\text { dermatológico }\end{array}$ & isolamento & $\mathrm{IFI}$ & $\begin{array}{c}\text { ELISA } \\
\text { leitura/cut off }\end{array}$ \\
\hline 1 & Dogue Alemão & bolsa escrotal & 1 & úlcera & positivo & NR & NR \\
\hline 2 & SRD & orelha direita & 1 & úlcera & positivo & $\mathrm{NR}$ & $0,167 / 0,142$ \\
\hline \multirow[t]{3}{*}{3} & SRD & focinho & 1 & úlcera & $\mathrm{NP}$ & & \\
\hline & & orelha esquerda & 2 & úlcera & positivo & NR & $0,529 / 0,142$ \\
\hline & & orelha esquerda & 3 & nódulo & $N P$ & & \\
\hline \multirow[t]{2}{*}{4} & SRD & bolsa escrotal & 1 & úlcera & positivo & NR & $0,26 / 0,09$ \\
\hline & & focinho & 1 & úlcera & NP & & \\
\hline \multirow[t]{3}{*}{5} & SRD & orelha direita & 5 & úlcero-crostosa & positivo* & 1:160 & $1,0 / 0,09$ \\
\hline & & orelha esquerda & 4 & úlcero-crostosa & positivo* & & \\
\hline & & focinho & 1 & úlcera & NP & & \\
\hline \multirow[t]{2}{*}{6} & SRD & orelha esquerda & 1 & úlcera & positivo & 1:40 & $0,29 / 0,09$ \\
\hline & & orelha direita & 1 & úlcera & positivo & & \\
\hline 7 & Dachshund & bolsa escrotal & 1 & úlcera & contaminado & $\mathrm{NR}$ & NR \\
\hline 8 & SRD & orelha direita & 1 & úlcera & negativo & NR & $0,12 / 0,09$ \\
\hline
\end{tabular}

$\widehat{S R D}=$ sem raça definida, $\mathrm{NR}=$ não reator, $\mathrm{NP}=$ não processado *pool de biópsias. 


\section{DISCUSSÃO}

No Estado do Rio de Janeiro, a ocorrência da LTA está associada a Leishmania (Viannia) braziliensis, onde cães domésticos desenvolvem a infecção com clínica similar ao homem ${ }^{13}$. O envolvimento desses animais em ciclos que se mantêm no domicílio ou peridomicílio, está associado principalmente aos ambientes alterados, favorecendo a adaptação de espécies vetoras ${ }^{8}$, como tem ocorrido nos últimos anos com a Lutzomyia intermedia ${ }^{15}$.

Neste estudo, sete dos oito animais clinicamente suspeitos para LTA residiam em áreas de loteamentos recentemente implantados no Município de MaricáRJ, próximo da mata residual (Serra da Tiririca). Não foi registrada a ocorrência de casos humanos nestas residências, embora, dois casos ativos tenham sido relatados na localidade de Inoã, área de procedência de cinco animais parasitologicamente positivos ${ }^{5}$.

A evolução clínica da LTA canina provocada pela $L$. braziliensis manifesta-se normalmente de forma crônica, sem comprometer o estado geral do animal, cujas lesões podem progredir em número e extensão6, evoluir para cura clínica espontânea com reativações posteriores ou, acometer tardiamente a mucosa nasal ${ }^{6}{ }^{14}$. Apesar deste estudo não ter como objetivo o acompanhamento dos animais, a auto-resolução de lesões foi observada em dois animais ( $n$ - 1 e 8) e a reativação no local original da lesão, no animal $n \times 1$, constatada após aproximadamente 2 anos. Este animal $(\mathrm{n}-1)$, apesar de ter sido parasitologicamente positivo não foi reagente aos testes sorológicos empregados.

O aspecto dermatológico observado nas lesões, não diferiu daqueles anteriormente descritos em cães naturalmente infectados. Também houve concordância quanto à localização das lesões, ocorrendo predomínio nas orelhas ${ }^{213}$.

Dos seis cães parasitologicamente positivos, dois tinham uma única lesão e quatro animais tinham lesões múltiplas. Em diversos trabalhos ${ }^{213}$, o encontro de lesões únicas tem prevalecido. No entanto, a multiplicidade de lesões também tem sido relatada, como descrito por Falqueto et al que relataram um caso canino com 10 úlceras $^{2}$.

No presente trabalho o cão $n \div 5$, inicialmente possuía cerca de 9 lesões, localizadas nas faces interna e externa das duas orelhas que confluíam. A presença de crostas, bem como o acometimento tardio da mucosa nasal (constatado em visita posterior), podem ser decorrência de uma infecção mais antiga. Tanto a extensão e multiplicidade das lesões, bem como o acometimento mucoso, poderiam explicar a forte reação sorológica encontrada nos dois testes. Tal fato também foi observado por Bray ${ }^{1}$ em humanos. Pirmez et $\mathrm{al}^{14}$, empregando a IFI em cães experimentalmente infectados na região da mucosa, demonstraram títulos superiores aos encontrados neste estudo. O cão № 6, apesar de também ter apresentado mais que uma lesão de grande extensão, demonstrou fraca reatividade nos mesmos testes.

A não reatividade do teste de IFI diante da positividade no ELISA dos cães de ํㅜ 2, 3, 4 e 8 pode ser explicada pela maior sensibilidade desse último. No entanto, quando aplicado em estudos epidemiológicos na LTA, a especificidade do ELISA pode tornar-se baixa, devido a ocorrência de reações inespecíficas ${ }^{17}$.

As chances de isolamento parasitário estão diretamente relacionadas às condições de coleta e cultivo, no entanto, a carga parasitária ${ }^{12}$ e o tempo de evolução ${ }^{7}$ nas lesões provocadas pela $L$. braziliensis podem também influenciar tal diagnóstico. Mengistu et $\mathrm{al}^{9}$, relataram ainda, uma irregulariedade na distribuição das amastigotas em lesão cutânea. Esses fatores podem justificar os resultados parasitológicos encontrados nos cães de $n^{\circ} 7$ e 8 .

Apenas cinco dos seis parasitas isolados foram caracterizados por critérios bioquímicos (isoenzimas), os quais demonstraram total homogeneidade com a cepa referência de Leishmania (Viannia) braziliensis, corroborando os achados de outros autores com isolados caninos de diferentes áreas do Município do Rio de Janeiro ${ }^{11}$.

Desta forma, alguns aspectos devem ser levados em consideração no diagnóstico da LTA canina, como a eventual presença de quadros dermatológicos incomuns, a ocorrência de problemas relacionados ao encontro do parasita in vitro e a possível variação na resposta humoral individual, já que nem sempre esta encontra-se associada à presença da lesão ativa. Assim, em cães com lesões sugestivas provenientes de áreas endêmicas ou daquelas cuja paisagem possa manter o ciclo parasitário, sugere-se o acompanhamento periódico do animal, objetivando a confirmação do diagnóstico da LTA.

\section{REFERÊNCIAS BIBLIOGRÁFICAS}

1. Bray RS. Immunodiagnosis of leishmaniasis. In: Chang KP, Bray RS (eds) Leishmaniasis. Elsevier Science Publishers B.V. Biomedicine Division p.117-182, 1985.

2. Falqueto A, Coura JR, Barros GC, Grimaldi G, Sessa PA, Carias VRD, Jesus AC, Alencar JTA. Participação do cão no ciclo de transmissão da leishmaniose tegumentar no município de Viana, estado do Espírito Santo, Brasil. Memórias do Instituto Oswaldo Cruz 81:155-163, 1986.
3. Fundação Nacional da Saúde. Vigilância Epidemiológica. Casos confirmados, segundo o período de diagnóstico e local de residência, por UF. Disponível em: http:// www.funasa.gov.br. Acesso em 10 de maio de 2002, Brasil, 1980-2000.

4. Lopes UG, Momen H, Grimaldi G, Marzochi MCA, Pacheco RS, Morel CM. Schizodeme and zymodeme characterization of Leishmania in the investigation of visceral and cutaneous leishmaniasis. Journal of Parasitology 70: 89-98, 1984. 
5. Madeira MF, Uchôa CMA, Magalhães CM, Duarte R, Macedo RM, Figlioulo L, Leal CA, Serra CMB. Avaliação da circulação da leishmaniose no município de Maricá-Rio de Janeiro. Um estudo de três anos. Revista da Sociedade Brasileira de Medicina Tropical 34 (supl I): 190-191, 2001.

6. Marco JD, Padilla AM, Diosque P, Fernández MM, Malchiodi EL, Basombrío MA. Force of infection and evolution of lesions of canine tegumentary leishmaniasis in northwestern Argentina. Memórias do Instituto Oswaldo Cruz 96:649-652, 2001.

7. Marzochi MCA. Doenças infecto-parasitárias: leishmanioses no Brasil - as leishmanioses tegumentares. Jornal Brasileiro de Medicina 63:82-104, 1992.

8. Marzochi MCA, Marzochi KB. Tegumentary and visceral leishmaniasis in Brazil - Emerging anthropozoonosis and possibilities for their control. Cadernos de Saúde Pública 10:359-375, 1994.

9. Mengistu G, Akuffo H, Fehninger TE, Negese Y, Nilsen R. Comparison of parasitological and immunological methods in the diagnosis of leishmaniasis in Ethiopia. Transaction of the Royal Society Tropical Medicine and Hygiene 86:154-157, 1992.

10. Momen H. Parasite characterization by zimodeme analysis. In: Morel CM (ed) Genes and Antigens of Parasites. A laboratory manual. Rio de Janeiro UNDP/World Bank/WHO-FINEP, CNPq, Fundação Oswaldo Cruz, Rio de Janeiro, p:11-120, 1984.

11. Oliveira-Neto MP, Pirmez C, Rangel E, Schubach A, Grimaldi Junior G. An outbreak of american cutaneous leishmaniasis (Leishmania braziliensis braziliensis) in a periurban area of Rio de Janeiro city, Brazil: clinical and epidemiological studies. Memórias do Instituto Oswaldo Cruz 83: 427-435, 1988.
12. Padilla AM, Marco DJ, Diosque $P$, Malchiodi E, Fernández M, Marinconz A, Basombrio MA. Leishmaniasis tegumentaria canina: evolutión de las lesiones y carga parasitaria. Medicina 59 (suppl III):54, 1999.

13. Pirmez C, Coutinho SG, Marzochi MCA, Nunes MP, Grimaldi Junior G. Canine american cutaneous leishmaniasis: a clinica and immunological study in dogs naturally infected with Leishmania braziliensis braziliensis in an endemic area of Rio de Janeiro, Brazil. American Journal of Tropical Medicine and Hygiene 38: 52-58, 1988.

14. Pirmez C, Marzochi MCA, Coutinho SG. Experimental canine mucocutaneous leishmaniasis (Leishmania braziliensis braziliensis). Memórias do Instituto Oswaldo Cruz 83: 145-151, 1988.

15. Rangel EF, Azevedo ACR, Andrade CA, Souza NA, Wermelinger ED. Studies on sandfly fauna (Diptera: Psychodidae) in a foci of cutaneous leishmaniasis in Mesquita, Rio de Janeiro state, Brazil. Memórias do Instituto Oswaldo Cruz 85: 39-45, 1990.

16. Reithinger R, Davies CR. Is the domestic dog (Canis familiaris) a reservoir host of American cutaneous leishmaniasis? A critical review of the current evidence. American Journal of Tropical Medicine and Hygiene 61: 530-541, 1999.

17. Uchôa CMA, Serra CMB, Duarte R, Magalhães CM, Macedo RM, Theophilo F, Fliglioulo L, Horta F, Madeira MF. Aspectos sorológicos e epidemiológicos da leishmaniose tegumentar americana canina em Maricá, Rio de Janeiro, Brasil. Revista da Sociedade Brasileira de Medicina Tropical 34: 563-568, 2001. 